CANCERS

\section{Dangers of Afiluence}

from our Cell Biology Correspondent

THE fig and prune producers of the world might do worse than start an advertising campaign with the slogan "a bowl a day keeps bowel cancer away" to judge from what Burkitt has to say in the current issue of Cancer (28, 3 ; 1971). Burkitt has, of course, spent many a long year trying to pin down environmental factors responsible for particular cancers, always arguing that the incidence of the disease can be significantly reduced more easily by eliminating or evading the causative agents than by trying to fathom out their mode of action and devise a cure. And his latest foray, this time into the epidemiology of cancer of the colon and rectum, leads him to the conclusion that the refined diets of the world's developed nations, in particular their lack of indigestible fibre, may, together with particular gut floras, be responsible for the comparatively high incidence of bowel cancers and other non-malignant and non-infectious diseases of the bowel in affluent societies.

The incidence of bowel cancer, which in most western countries is second only to the incidence of lung cancer, is correlated with the stage of economic development, and Burkitt claims that the age adjusted incidence of this disease is ten-fold greater in Western Europe and North America than in most African countries. Epidemiologists when faced with such findings frequently resort to the incantation "dietary differences" and leave it at that with the real problem, the identification of the crucial dietary components, unsolved. Burkitt to his credit has given hostages to fortune by suggesting what the crucial factors may be, namely the transit time of the stools through the bowel and the nature of the bowel flora.

By recruiting the help of African and English boarding school boys, who were provided with radio-opaque plastic pellets as a condiment for their school meals, he and his collaborators have shown that there is a clear inverse relationship between the transit time and bulk of the stools and the fibre content of the food eaten-the more fibre the shorter the transit time. African boys passed 80 per cent of the plastic pellets in $45 \mathrm{~h}$ whereas their English counterparts took 89 h. Burkitt suggests therefore that carcinogens produced by the bacterial flora in the bowels of westerners, whose foetid stools contrast markedly with the odourless stools of Africans, are held at high concentrations for prolonged periods in contact with the bowel mucosa with the net result of an increased incidence of bowel cancer in western populations. Like a prophet in the wilderness Burkitt writes: "In view of the evidence, it seems justifiable to issue a warning against the removal of so much of the unadsorbable fibre from our food, and the associated over ingestion of refined carbohydrate".

Another and related warning was published recently by Hill, Goddard and Williams (Lancet, i, 472; 1971) who suggest that differences in fat consumption together with differences in gut flora may account for the geographical variations in the incidence of breast cancer. It is well known that mammary carcinoma is far more prevalent among North American and North West European women than among African, Asian and South American women, and surveys of the Japanese populations in Japan, Hawaii and the West Coast of the United States show that differences in the incidence of breast cancer depend on geographic rather than genetic factors. Dietary differences have been discussed, and Wynder, for example, has postulated that the consumption of large amounts of fat may affect hormone production and/or storage in adipose tissue and thus be responsible for an increased incidence of breast cancer.

Hill and his colleagues now suggest an alternative mechanism. They have shown that people eating high fat diets have in their guts more active and strictly anaerobic bacteria than people eating low fat diets. Moreover these bacteria are able to produce oestradiol, oestrone and several other steroid sex hormones. If one or more of these steroids is carcinogenic or acts synergistically and stimulates the growth of tumour cells, anaerobic gut bacteria may be the link between high fat diets and a high incidence of breast cancer, and for that matter colonic cancer. If the much discussed expansion of cancer research materializes, the distributors of the dollars might do worse than promote further investigations of the metabolic activities of gut bacteria.

\title{
Liquid Drops in Shock Tubes
}

The photograph below shows some of the bizarre shapes that drops of water can take up in a shock tube. The drops are falling through a flow that is from left to right, and the aerodynamic and surface tension forces involved have given the drops an umbrella-shaped canopy. The photograph is one of several in next Monday's Nature Physical Science that have been obtained by P. G. Simpkins of Bell Telephone Laboratories, Whippany, New Jersey, illustrating the successive deformations that drops underyo when exposed to a sudden increase in the external flow.

The crucial parameter in work of this nature is the ratio of the force due to aerodynamic pressure to the force due to surface tension-the Weber number -and the photograph was taken at a Weber number of 45 . But the region in which Simpkins is interested has a lower limit at a Weber number of about 7.3-above this critical value the nonuniform distribution of surface pressure exerted on the drops causes deformations which eventually lead to the bursting of the drops.

The steps in this process, as charted by Simpkins, are as follows. At a Weber number of 19 drops that initially had a diameter of $1 \mathrm{~mm}$ are flattened by the flow into thin disks. The centres of the disks then blow out in the direction of the external flow, so that the drop takes on the appearance of a bag supported by a liquid annulus. Simpkins presents graphs showing how the distortion proceeds, and discusses the deviations in behaviour from those predicted by simple theory.

The deviations from expected behaviour become more severe at higher Weber numbers. Umbrellashaped distortion of the drops like that in the photograph occurs at Weber numbers above twenty. The mechanism by which the drops form a narrow stem and subsequently a canopy is, however, unknown.

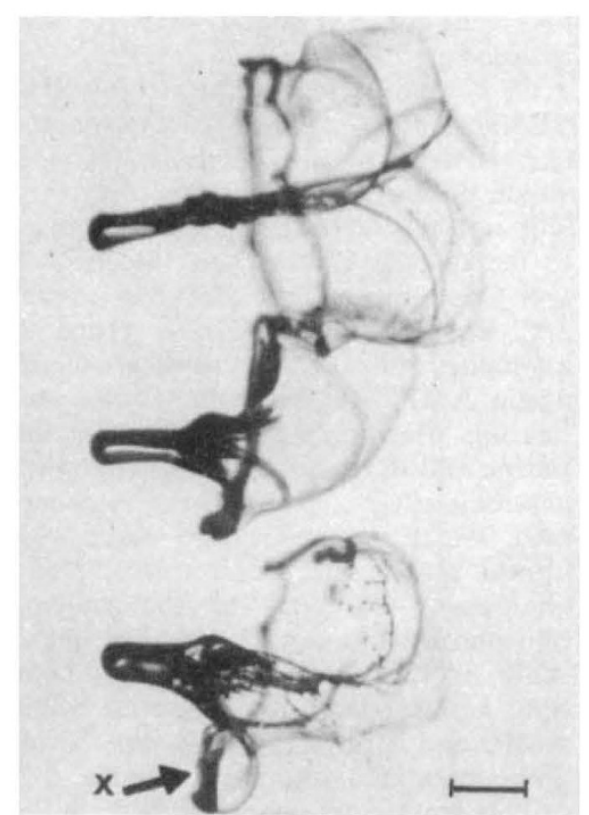

Drops in a shock tube at a Weber number of $45,1,660 \mu \mathrm{s}$ after the start of the flow. The flow is from left to right and the bar represents $1 \mathrm{~mm}$. The arrow points to a satellite drop which is showing the bag response. 